\title{
FORMAS DE FÓSFORO NO SOLO SOB LEGUMINOSAS FLORESTAIS, FLORESTA SECUNDÁRIA E PASTAGEM NO NORTE FLUMINENSE(1)
}

\author{
Francisco Costa Zaia ${ }^{(2)}$, Antonio Carlos da Gama-Rodrigues ${ }^{(3)} \&$ \\ Emanuela Forestieri da Gama-Rodrigues ${ }^{(3)}$
}

\begin{abstract}
RESUMO
Em solos muito intemperizados, a disponibilidade de $\mathrm{P}$ para as plantas está estreitamente associada às formas de $\mathrm{P}$ orgânico, que tem o seu conteúdo alterado por influência do tipo de cobertura vegetal. O objetivo deste trabalho foi estimar os teores de fósforo de compostos orgânicos (Po-total, Po lábil e P microbiano do solo), em plantios de Acacia auriculiformis (acácia) e Mimosa caesalpiniifolia (sabiá), em floresta secundária e em pastagem. Os teores médios ponderados de Po total e Po lábil, pelo teor de argila, foram de $375 \mathrm{mg} \mathrm{kg}^{-1}$ de $P$ na argila e de $40,2 \mathrm{mg} \mathrm{kg}^{-1}$ de $\mathrm{P}$ na argila, respectivamente. Os solos sob acácia e sabiá apresentaram maior teor relativo de Po (total e lábil) do que os solos sob pastagem e capoeira. O Po representou de 22,6 a $39,6 \%$ do $P$ total extraído dos solos das coberturas estudadas. Em relação ao P lábil total, o Po lábil representou, em média, 67,1 \%. Para o P microbiano, o maior teor foi encontrado no solo sob sabiá, seguido da floresta secundária, acácia e pastagem. Contudo, foi na capoeira que houve a maior eficiência da biomassa microbiana do solo em imobilizar $\mathbf{P}$. O teor de $\mathbf{P}$ disponível por Mehlich-1 correlacionou-se positivamente com o teor de $\mathbf{C}$ e o de $\mathbf{P}$ da biomassa microbiana. Houve estreita correlação positiva entre o $\mathbf{P}$ microbiano e o C microbiano.
\end{abstract}

Termos de indexação: disponibilidade, fósforo orgânico, fósforo lábil, biomassa microbiana, Mata Atlântica.

\footnotetext{
(1) Parte da Tese de Mestrado do primeiro autor, apresentada ao Curso de Pós-Graduação em Produção Vegetal, Universidade Estadual do Norte Fluminense - CCTA/UENF. Recebido para publicação em junho de 2007 e aprovado em fevereiro de 2008.

${ }^{(2)}$ Doutorando do Curso de Produção Vegetal, Universidade Estadual Norte Fluminense - UENF. Av. Alberto Lamego 2000, Parque Califórnia, CEP 28013-602 Campos dos Goytacazes (RJ). Email: zaia@uenf.br

(3) Professor Associado do Laboratório de Solos do Centro de Ciências e Tecnologias Agropecuárias (CCTA), UENF. E-mails: tonygama@uenf.br; emanuela@uenf.br
} 


\title{
SUMMARY: SOIL PHOSPHORUS FORMS UNDER LEGUMINOUS TREE SPECIES, SECONDARY FOREST AND PASTURE IN NORTHERN RIO DE JANEIRO STATE, BRAZIL
}

\begin{abstract}
In highly weathered tropical soils phosphorus $(P)$ bioavailability to plants is closely associated with soil organic $P$ forms, which in turn are influenced by the vegetation cover. The aim of this study was to evaluate total organic $P(P o)$, labile $P$ fractions and microbial biomass $P$ in soils under Acacia auriculiformis and Mimosa caesalpiniifolia stands, secondary forest and pasture. The clay-adjusted average content of total Po and labile Po were $375 \mathrm{mg} \mathrm{kg}^{-1} \mathrm{P}$ and $40.2 \mathrm{mg} \mathrm{kg}^{-1}$, respectively. The Po contents (total and labile) in soils under Acacia auriculiformis and Mimosa caesalpiniifolia were higher than under pasture and secondary forest soils. The proportion of Po ranged from 22.6 to $39.6 \%$ of total extracted $P$ in soils under the vegetation cover types studied. The labile Po represented about $67.1 \%$ of total labile $P$. The greatest amount of soil microbial biomass $P(M B P)$ was observed under Mimosa caesalpiniifolia, followed by secondary forest, Acacia auriculiformis and pasture. However, the soil microbial biomass of the secondary forest was the most efficient regarding $P$ immobilization ( $M B P /$ total Po ratio). Available $P$ was positively correlated with soil microbial biomass $C$ and microbial $P$, and microbial $P$ was positively related to microbial $C$.
\end{abstract}

Index terms: available $P$, labile $P$, organic $P$, microbial biomass, Atlantic Forest.

\section{INTRODUÇÃO}

Nos solos tropicais muito intemperizados e argilosos, a capacidade de fixação de P é elevada, reduzindo sua disponibilidade às plantas. Desse modo, o solo compete com a planta pelo elemento, deixando de ser fonte para tornar-se dreno. Assim, em solos dreno praticamente não deve haver contato do $\mathrm{P}$ ciclado com a fase mineral desses solos. A planta absorveria diretamente do que mineralizasse do substrato orgânico (serapilheira) ou da fase orgânica (P orgânico no solo) sem dar chances ao substrato mineral do solo de envolver-se no equilíbrio existente (Novais \& Smyth, 1999). Nesse sentido, a disponibilidade de $P$ constitui o fator nutricional mais limitante à produção vegetal nos solos intemperizados. Nesses solos, portanto, o ciclo bioquímico (ciclo biológico) é mais relevante que o ciclo geoquímico (Chen et al., 2003). A proporção de $\mathrm{P}$ orgânico no solo tende a aumentar com o avanço do intemperismo (Cross \& Schlesinger, 1995; Gressel \& McColl, 1997; Grierson et al., 2004).

A participação do $\mathrm{P}$ orgânico pode variar de 3 a $90 \%$ do teor de $\mathrm{P}$ total, ou de 1 a $3 \%$ da matéria orgânica do solo (Siqueira \& Moreira, 2001), podendo corresponder à maior parte do teor de $\mathrm{P}$ disponível no solo (Guerra et al., 1996; Cunha et al., 2007). Além disso, o P orgânico é adsorvido preferencialmente em relação ao $\mathrm{P}$ inorgânico, tendo assim um efeito benéfico indireto, ao aumentar a disponibilidade do $\mathrm{P}$ mineral no solo (Cantarella et al., 1992).

A dinâmica do $\mathrm{P}$ orgânico está estreitamente associada à dinâmica da matéria orgânica do solo (Gressel \& McColl, 1997; Solomon et al., 2002; Cunha et al., 2007). Assim, agroecossistemas acumuladores de matéria orgânica e de baixos insumos, como são os sistemas agroflorestais e florestais (Fontes, 2006; Gama-Rodrigues et al., 2007), seriam capazes de manter adequadamente a disponibilidade de $\mathrm{P}$ para as plantas (Gama-Rodrigues et al., 1999; Szott \& Melendez, 2001; Lehmann et al., 2001), mediante a mineralização de $\mathrm{P}$ orgânico (Grierson et al., 1999; George et al., 2002; Comerford et al., 2006).

Além disso, em solos tropicais, o $\mathrm{P}$ orgânico pode ser grandemente afetado pelo tipo de cobertura florestal ou agroflorestal, alterando a disponibilidade de P (Szott \& Mellendez, 2001; Solomon et al., 2002; Cunha et al., 2007). Assim, a caracterização do Po tem importância fundamental na compreensão do ciclo do $\mathrm{P}$ em solos de avançado estádio de intemperismo.

O objetivo deste trabalho foi verificar a variação nos teores de $\mathrm{P}$ de compostos orgânicos Po total (Po), Po lábil e P microbiano do solo, em plantios de duas leguminosas florestais, acácia e sabiá, em floresta secundária e em pastagem.

\section{MATERIAL E MÉTODOS}

O trabalho foi realizado na Fazenda Carrapeta, no município de Conceição de Macabu, RJ. O clima da região, pela classificação de Köppen, é do tipo Am, quente e úmido. De acordo com informações locais, a temperatura está por volta de $26^{\circ} \mathrm{C}$, e a precipitação média anual é de $1.400 \mathrm{~mm}$, com período chuvoso entre outubro e março e seco entre junho e setembro. $\mathrm{O}$ 
solo foi classificado como Latossolo Vermelho-Amarelo, Tb caulinítico (Comunicado pessoal de Prof. Doracy Pessoa Ramos), em relevo ondulado, com declividade em torno de $35 \mathrm{~cm} \mathrm{~m}^{-1}$.

A área experimental constituiu-se de quatro coberturas vegetais em parcelas de $1.500 \mathrm{~m}^{2}$ $(75 \times 20 \mathrm{~m})$. As parcelas experimentais de cada cobertura foram dispostas adjacentes uma da outra, na mesma cota de altitude. As coberturas vegetais foram constituídas de plantios puros das espécies arbóreas Acacia auriculiformis (acácia) e Mimosa caesalpiniifolia (sabiá), inoculadas com estirpes selecionadas de bactérias fixadoras de $\mathrm{N}_{2}$ e fungos micorrízicos. O plantio das espécies arbóreas foi realizado em dezembro de $1998 \mathrm{com}$ mudas de $30 \mathrm{~cm}$ de altura e apresentando ótima nodulação e micorrização. O espaçamento utilizado foi de $3 \times 2 \mathrm{~m}$, em covas de $20 \times 20 \times 20 \mathrm{~cm}$, às quais foram adicionados $150 \mathrm{~g}$ de superfosfato simples, $10 \mathrm{~g}$ de cloreto de K e 10 g de FTE-BR12. As outras duas coberturas vegetais, adjacentes aos plantios puros, utilizados como referências, ambas com aproximadamente 40 anos de idade, foram um pasto degradado, que representa a vegetação anterior ao plantio das espécies arbóreas, com predomínio de capim-gordura (Melinis minutiflora), grama-pernambuco (Paspalum maritimum) e sapê (Imperata brasiliensis); e um fragmento florestal de Mata Atlântica em sucessão secundária, com espécies em diferentes estádios sucessionais (capoeira). A pastagem foi considerada degradada por apresentar baixa disponibilidade de forragem, em decorrência do manejo inadequado com uso indiscriminado de fogo e superpastejo e sem adição de fertilizantes fosfatados. A escolha da capoeira como uma das coberturas de referência trata-se de uma das estratégias utilizadas para avaliar alterações do solo em decorrência do tipo de uso e de técnicas de manejo por meio da comparação de características do solo manejado com a do solo não manejado, sob vegetação natural (Barros \& Comerford, 2002).

Para caracterizar a fertilidade do solo, os teores de Po lábil e Po total e os teores de $\mathrm{C}$ e $\mathrm{P}$ da biomassa microbiana em cada cobertura vegetal, foram coletadas quatro amostras compostas, sendo cada uma constituída de quinze amostras simples, ao acaso, nas entrelinhas de plantio, na camada de $0-5 \mathrm{~cm}$, em outubro de 2003. A composição granulométrica e os atributos químicos foram determinados de acordo com Embrapa (1997), com exceção do C orgânico (CO) dosado por oxidação com $\mathrm{K}_{2} \mathrm{Cr}_{2} \mathrm{O}_{7} 1,25 \mathrm{~mol} \mathrm{~L}^{-1} \mathrm{em}$ meio ácido (Anderson \& Ingram, 1996). Foram também coletadas amostras de solo na camada de $0-10 \mathrm{~cm}$ para determinação dos teores das frações de Po e $\mathrm{P}$ de compostos inorgânicos $(\mathrm{Pi})$.

O Po total foi obtido por meio do método de extração seqüencial (Bowman, 1989), e o Po lábil, pelo método de extração com $\mathrm{NaHCO}_{3} 0,5 \mathrm{~mol} \mathrm{~L}^{-1}$ (Bowman \& Cole, 1978). O Pi foi determinado após clarificação dos extratos com carvão ativo (Guerra et al., 1996).
O teor de Pi nos extratos ácidos e alcalinos foi determinado pelo método de Murphey \& Riley (1962). $\mathrm{O}$ método da fumigação-extração foi utilizado para estimar o C (Vance et al., 1987) e o P (Brooks et al., 1984) da biomassa microbiana do solo.

Os teores de P disponível (Mehlich-1), das frações de $\mathrm{P}$ inorgânico, $\mathrm{P}$ orgânico, $\mathrm{P}$ inorgânico + $\mathrm{P}$ orgânico, de $\mathrm{P}$ da biomassa microbiana, de $\mathrm{C}$ orgânico e de $\mathrm{C}$ da biomassa microbiana, na camada de $0-5 \mathrm{~cm}$, foram corrigidos pelo teor de argila do solo de cada cobertura vegetal. Os resultados foram expressos em $\mathrm{mg} \mathrm{kg}^{-1}$ de argila, à exceção para $\mathrm{C}$ orgânico expresso em $\mathrm{g} \mathrm{kg}^{-1}$ de argila.

Os dados foram submetidos à análise de variância como em delineamento inteiramente casualizado com quatro repetiç̃es, nas análises dos atributos químicos e físicos do solo, do $\mathrm{P}$ orgânico total e lábil e $\mathrm{P}$ inorgânico do solo, e do $\mathrm{C}$ e $\mathrm{P}$ da biomassa microbiana do solo. Adotou-se o teste $\mathrm{F}$ a 5 \%. De maneira complementar, utilizou-se, para comparação de médias, o teste de Tukey a $5 \%$. Cada cobertura florestal foi considerada um tratamento de efeito-fixo, a exemplo do procedimento empregado por Lugo et al. (1990) e GamaRodrigues et al. (1999). Em cada cobertura vegetal, o mesmo procedimento estatístico foi adotado na comparação entre as camadas de amostragem para os teores das frações de $\mathrm{P}$ inorgânico, $\mathrm{P}$ orgânico e $\mathrm{P}$ inorgânico + P orgânico do solo.

Foram estabelecidas correlações de Pearson a $5 \%$ de probabilidade $(n=16)$ entre as diferentes frações de $\mathrm{P}$ e alguns atributos químicos do solo.

\section{RESULTADOS E DISCUSSÃO}

\section{Camada de amostragem}

Os teores das frações de fósforo inorgânico (Pi), fósforo orgânico (Po) e P total ( $\mathrm{Pi}+\mathrm{Po})$ na camada de $0-5 \mathrm{~cm}$ foram significativamente maiores do que na camada $0-10 \mathrm{~cm}$ em todas as coberturas vegetais (Quadro 1). Estes resultados indicam que camadas de amostragem superiores a $0-5 \mathrm{~cm}$ proporcionariam "efeito de diluição" na determinação das frações de $\mathrm{P}$ em solos florestais e de pastagens. Nessas coberturas vegetais, a ciclagem biogeoquímica de $\mathrm{P}$ seria decorrente da deposição de resíduos da parte aérea na superfície do solo (Silva, 2005), no qual a incorporação desses resíduos mediante o processo de decomposição dar-se-ia predominantemente nos primeiros centímetros do perfil do solo. Além disso, na camada de $0-5 \mathrm{~cm}$, também ocorreria grande presença de raízes finas e radicelas (Gama-Rodrigues \& CadimaZevallos, 1991; Gama-Rodrigues et al., 1999).

\section{Cobertura vegetal}

O solo sob capoeira apresentou características químicas típicas de condições de baixa fertilidade de 
nutrientes, acidez muito elevada e com presença de alto teor de $\mathrm{Al}^{3+}$ no complexo de troca (Quadro 2). As coberturas de leguminosas promoveram certa melhoria na fertilidade do solo, indicada pela menor acidez e maiores valores de soma de bases. Contudo, os teores de $\mathrm{CO}$ e de $\mathrm{P}$ disponível (Mehlich-1) pouco variaram entre as coberturas.

Entretanto, considerando o teor de argila, que variou significativamente entre as coberturas vegetais, verificou-se que os solos sob as leguminosas florestais e a pastagem apresentaram maiores teores ponderados, de acordo com o teor de argila, de CO e P disponível do que o solo sob capoeira, que apresentou o maior teor de argila (Quadro 1). Para esses dois atributos químicos, as leguminosas também apresentaram teores superiores aos da pastagem.
O teor médio ponderado de Po total nos solos sob as coberturas vegetais foi de $375 \mathrm{mg} \mathrm{kg}^{-1}$ de $\mathrm{P}$ na argila (Quadro 3). As leguminosas florestais apresentaram maiores teores de Po total do que a pastagem e a capoeira. Situação análoga foi verificada para o teor de Po lábil; por outro lado, não houve variação significativa entre a pastagem e a capoeira.

O Pi predominou na composição do $\mathrm{P}$ total $(\mathrm{Pi}+\mathrm{Po})$ nos solos sob todas as coberturas vegetais. O Po representou de 22,6 a 39,6 \% do $\mathrm{P}$ total extraído. O solo sob capoeira apresentou a menor relação $\mathrm{Po} /$ $\mathrm{P}$ total, enquanto a maior participação do Po na composição do P total foi no solo sob acácia (Quadro 3). Cunha et al. (2007) verificaram para diversos solos florestais e de pastagens que o Po representou de 14,6 a $36,9 \%$ do $\mathrm{P}$ total extraído.

Quadro 1. Teores das frações de fósforo inorgânico (Pi), orgânico (Po) e inorgânico + orgânico (Pi + Po) nas formas de $\mathrm{P}$ total e lábil do solo, nas camadas de 0-5 e de 0-10 cm, sob diferentes coberturas vegetais na região Norte Fluminense

\begin{tabular}{|c|c|c|c|c|c|c|}
\hline \multirow[b]{2}{*}{ Camada } & \multicolumn{2}{|c|}{$\mathbf{P i}$} & \multicolumn{2}{|c|}{ Po } & \multicolumn{2}{|c|}{$\mathbf{P i}+\mathbf{P o}$} \\
\hline & Total & Lábil & Total & Lábil & Total & Lábil \\
\hline \multirow[t]{2}{*}{$\mathrm{cm}$} & \multicolumn{6}{|c|}{$-\mathrm{mg} \mathrm{kg}^{-1}$} \\
\hline & \multicolumn{6}{|c|}{ Acácia } \\
\hline $0-5$ & $183,29 \mathrm{a}$ & $4,78 \mathrm{a}$ & $119,99 \mathrm{a}$ & $10,13 \mathrm{a}$ & $303,28 \mathrm{a}$ & $14,90 \mathrm{a}$ \\
\hline \multirow[t]{2}{*}{$0-10$} & $174,58 \mathrm{~b}$ & $2,38 \mathrm{~b}$ & $90,30 \mathrm{~b}$ & $7,35 \mathrm{~b}$ & $264,87 \mathrm{~b}$ & $9,73 \mathrm{~b}$ \\
\hline & \multicolumn{6}{|c|}{ Sabiá } \\
\hline $0-5$ & $253,60 \mathrm{a}$ & $4,80 \mathrm{a}$ & $79,05 \mathrm{a}$ & $9,02 \mathrm{a}$ & $332,95 \mathrm{a}$ & $13,82 \mathrm{a}$ \\
\hline \multirow[t]{2}{*}{$0-10$} & $196,04 \mathrm{~b}$ & $2,92 \mathrm{~b}$ & $69,42 \mathrm{~b}$ & $6,41 \mathrm{~b}$ & $265,46 \mathrm{~b}$ & $9,33 \mathrm{~b}$ \\
\hline & \multicolumn{6}{|c|}{ Pasto } \\
\hline $0-5$ & $269,13 \mathrm{a}$ & $4,66 \mathrm{a}$ & $85,29 \mathrm{a}$ & $8,48 \mathrm{a}$ & $354,42 \mathrm{a}$ & $13,14 \mathrm{a}$ \\
\hline \multirow[t]{2}{*}{$0-10$} & $247,89 \mathrm{~b}$ & $3,25 \mathrm{~b}$ & $67,05 \mathrm{~b}$ & $6,63 \mathrm{~b}$ & $314,93 \mathrm{~b}$ & $9,87 \mathrm{~b}$ \\
\hline & \multicolumn{6}{|c|}{ Capoeira } \\
\hline $0-5$ & $226,20 \mathrm{a}$ & $4,38 \mathrm{a}$ & $66,08 \mathrm{a}$ & $11,18 \mathrm{a}$ & $292,28 \mathrm{a}$ & $15,56 \mathrm{a}$ \\
\hline $0-10$ & $166,30 \mathrm{~b}$ & $2,76 \mathrm{~b}$ & $40,41 \mathrm{~b}$ & $10,07 \mathrm{~b}$ & $206,71 \mathrm{~b}$ & $12,83 \mathrm{~b}$ \\
\hline
\end{tabular}

Médias seguidas por letras iguais, dentro de cada cobertura vegetal, na coluna, não diferem entre si pelo teste $\mathrm{F}$ a $5 \%$.

Quadro 2. Características físicas e químicas do solo, na camada de 0-5 cm, sob diferentes coberturas vegetais na região Norte Fluminense

\begin{tabular}{|c|c|c|c|c|c|c|c|c|c|c|}
\hline Cobertura & Areia & Silte & Argila & $\mathrm{pH} \mathrm{H}_{2} \mathrm{O}$ & $\mathbf{P}$ & $\mathbf{P} \mathbf{p}^{(1)}$ & $\mathrm{Al}^{3+}$ & SB & $\mathrm{CO}$ & $\mathrm{CO}_{\mathrm{p}}{ }^{(2)}$ \\
\hline & \multicolumn{3}{|c|}{$-\mathrm{g} \mathrm{kg}^{-1}$} & & \multicolumn{2}{|c|}{$\mathrm{mg} \mathrm{kg}^{-1}$} & \multicolumn{2}{|c|}{$-\mathrm{mmol}_{\mathrm{c}} \mathrm{kg}^{-1}-$} & \multicolumn{2}{|c|}{$-\mathrm{g} \mathrm{kg}^{-1}$} \\
\hline Acácia & $640 \mathrm{~b}$ & $140 \mathrm{~b}$ & $220 \mathrm{c}$ & $5,1 \mathrm{a}$ & $5,3 \mathrm{a}$ & $24,1 \mathrm{a}$ & $2,0 \mathrm{c}$ & $39,1 \mathrm{a}$ & $31,9 \mathrm{a}$ & $145 \mathrm{~b}$ \\
\hline Sabiá & $660 \mathrm{a}$ & $160 \mathrm{a}$ & $180 \mathrm{~d}$ & $4,9 \mathrm{a}$ & $4,4 \mathrm{~b}$ & 24,4 a & $2,0 \mathrm{c}$ & $36,0 \mathrm{a}$ & $31,0 \mathrm{a}$ & $172 \mathrm{a}$ \\
\hline Pasto & $600 \mathrm{c}$ & $140 \mathrm{~b}$ & $260 \mathrm{~b}$ & $4,9 \mathrm{a}$ & $3,9 \mathrm{~b}$ & $18,8 \mathrm{~b}$ & $4,0 \mathrm{~b}$ & $25,6 \mathrm{~b}$ & $29,4 \mathrm{~b}$ & $113 \mathrm{c}$ \\
\hline Capoeira & $570 \mathrm{~d}$ & $80 \mathrm{c}$ & $350 \mathrm{a}$ & $4,3 \mathrm{~b}$ & $4,1 \mathrm{~b}$ & $12,3 \mathrm{c}$ & $10,0 \mathrm{a}$ & $15,4 \mathrm{c}$ & $31,2 \mathrm{a}$ & $89 \mathrm{~d}$ \\
\hline
\end{tabular}

(1) Pp: fósforo ponderado de acordo com o teor de argila ( $\mathrm{mg} \mathrm{kg}^{-1}$ de $\mathrm{P}$ na argila). ${ }^{(2)} \mathrm{CO}_{\mathrm{p}}$ : carbono orgânico ponderado de acordo com o teor de argila ( $\mathrm{g} \mathrm{kg}^{-1}$ de CO na argila). Médias seguidas por letras iguais, na coluna, não diferem entre si pelo teste de Tukey a $5 \%$. 
Quadro 3. Teores das frações de P inorgânico (Pi), orgânico (Po) e Pi + Po nas formas de P lábil e total ponderados de acordo com o teor de argila, na camada de 0-5 $\mathrm{cm}$ do solo, sob diferentes coberturas vegetais na região Norte Fluminense

\begin{tabular}{|c|c|c|c|c|c|c|}
\hline \multirow{2}{*}{ Cobertura } & \multicolumn{2}{|c|}{$\mathbf{P i}$} & \multicolumn{2}{|c|}{ Po } & \multicolumn{2}{|c|}{$\mathbf{P i}+\mathbf{P o}$} \\
\hline & Total & Lábil & Total & Lábil & Total & Lábil \\
\hline Acácia & $833 c(60,4)^{(1)}$ & $21,7 \mathrm{~b}(32,1)^{(2)}$ & $545 \mathrm{a}(39,6)^{(1)}$ & $46,0 \mathrm{~b}(67,9)^{(2)}$ & $1.378 \mathrm{~b}$ & $67,7 \mathrm{~b}$ \\
\hline Sabiá & $1.409 \mathrm{a}(76,2)$ & $26,7 \mathrm{a}(34,8)$ & $439 \mathrm{~b}(23,8)$ & $50,1 \mathrm{a}(65,2)$ & $1.848 \mathrm{a}$ & $76,8 \mathrm{a}$ \\
\hline Pasto & $1.035 \mathrm{~b}(75,9)$ & $17,9 \mathrm{c}(35,4)$ & $328 \mathrm{c}(24,1)$ & $32,6 \mathrm{c}(64,6)$ & $1.363 \mathrm{~b}$ & $50,5 \mathrm{c}$ \\
\hline Capoeira & $646 \mathrm{~d}(77,4)$ & $12,5 \mathrm{~d}(28,1)$ & $189 \mathrm{~d}(22,6)$ & $32,0 \mathrm{c}(71,9)$ & $835 \mathrm{c}$ & $44,5 \mathrm{~d}$ \\
\hline Média geral & $981(72,3)$ & $19,7(32,9)$ & $375(27,7)$ & $40,2(67,1)$ & 1.356 & 59,9 \\
\hline
\end{tabular}

(1) Percentual em relação a Pi + Po total. ${ }^{(2)}$ Percentual em relação a Pi + Po lábil. Médias seguidas por letras iguais, na coluna, não diferem entre si pelo teste de Tukey a $5 \%$.

O balanço entre Pi lábil e Po lábil em relação ao $\mathrm{P}$ lábil total apresentou pequena diferença entre as coberturas vegetais (Quadro 3). A fração Po lábil foi maior do que Pi lábil, ao contrário do balanço Pi e Po em relação ao $\mathrm{P}$ total. No solo sob capoeira, $71,9 \%$ do P lábil foi constituído por Po lábil, enquanto, para as demais coberturas, variou de 64,6 a $67,9 \%$. O predomínio de Po lábil em relação ao Pi lábil (Quadro 3), encontrado também por Guerra et al. (1996) e Cunha et al. (2007), indicaria que a disponibilidade de P, em curto prazo, não seria completamente acessada por determinações de P disponível (Novais \& Smyth, 1999). Em solos de avançado estádio de intemperismo, o P disponível estaria estreitamente relacionado com as frações do Po (Tiessen et al., 1984; Beck \& Sanches, 1994; Cunha et al, 2007).

Houve diferença significativa entre as coberturas vegetais para o $\mathrm{P}$ da biomassa microbiana (Quadro 4). $\mathrm{O}$ solo sob sabiá apresentou o maior valor de $\mathrm{P}$ microbiano, seguido da capoeira, acácia e pastagem. Dentre as leguminosas, o $\mathrm{P}$ microbiano do sabiá foi 2,3 vezes superior ao da acácia. Para o $\mathrm{C}$ da biomassa microbiana, também houve diferença significativa entre as coberturas vegetais, no qual o maior valor ocorreu no solo sob sabiá e o menor no solo sob acácia, não se verificando distinção significativa entre a pastagem e a capoeira.

A maior eficiência da biomassa microbiana do solo em imobilizar C e P ocorreu no sabiá e na capoeira, respectivamente, indicada pelos maiores valores da relação de $\mathrm{C}$ microbiano para $\mathrm{C}$ orgânico do solo $(\mathrm{Cbm} /$ $\mathrm{CO})$ e da relação de $\mathrm{P}$ microbiano para Po total ( $\mathrm{Pbm} /$ Po total) (Quadro 4). A maior proporção de Po mineralizável, indicada pela relação Po lábil/Po total, também ocorreu nos solos sob capoeira (16,9\%) e sabiá (11,4 \%), seguido do pasto (10,1\%) e da acácia $(8,4 \%)$.

Não houve correlação significativa entre as frações de Po (total, lábil e microbiano) e o CO do solo.
Aparentemente, o grau de influência do tipo de vegetação sobre a dinâmica do Po não estaria estreitamente associado aos níveis de alterações do teor de CO no solo. Guerra et al. (1996) também não encontraram correlação significativa entre $\mathrm{CO}$ e Po total em Latossolos sob pastagens, mas, sim, em Argissolos para as frações de Po total e lábil. Correlações positivas dessas duas frações de Po com o CO foram reportadas por Cunha et al. (2007) em Latossolos e Cambissolos sob diversos tipos florestais e pastagens na região montana do Norte Fluminense. Esses autores também utilizaram os mesmos métodos de determinação de Po do presente trabalho.

Houve correlação positiva entre $\mathrm{P}$ microbiano e $\mathrm{C}$ da biomassa microbiana $(r=0,578 ; \mathrm{p}<0,05)$. Isto indica que ambos os elementos foram estimados da mesma fração da biomassa microbiana do solo. Por

\section{Quadro 4. Teores das frações de $\mathbf{P}$ da biomassa microbiana $(\mathrm{Pbm})$ e de $\mathrm{C}$ da biomassa microbiana $(\mathrm{Cbm})$, relações de $\mathrm{C}$ microbiano para $\mathrm{C}$ orgânico $(\mathrm{Cbm} / \mathrm{CO})$ e de $\mathrm{P}$ microbiano para Po total ( $\mathrm{Pbm} / \mathrm{Pot})$ ponderados de acordo com o teor de argila, na camada de $0-5 \mathrm{~cm}$ do solo, sob diferentes coberturas vegetais na região Norte Fluminense}

\begin{tabular}{|c|c|c|c|c|}
\hline Cobertura & $\mathrm{Pbm}$ & $\mathrm{Cbm}$ & $\mathrm{Cbm} / \mathrm{CO}$ & $\mathrm{Pbm} / \mathrm{Po}$ \\
\hline & \multicolumn{2}{|c|}{$-\mathrm{mg} \mathrm{kg}^{-1}$} & — & - \\
\hline Acácia & $15,9 \mathrm{c}$ & $649 \mathrm{c}$ & $0,45 \mathrm{c}$ & $2,92 \mathrm{c}$ \\
\hline Sabiá & 36,3 a & $1.837 \mathrm{a}$ & $1,07 \mathrm{a}$ & $8,27 \mathrm{~b}$ \\
\hline Pasto & $7,5 \mathrm{~d}$ & $859 \mathrm{~b}$ & $0,76 \mathrm{~b}$ & $2,29 \mathrm{~d}$ \\
\hline Capoeira & $30,1 \mathrm{~b}$ & $892 \mathrm{~b}$ & $1,00 \mathrm{a}$ & $15,93 \mathrm{a}$ \\
\hline
\end{tabular}

Médias seguidas por letras iguais, na coluna, não diferem entre si pelo teste de Tukey a $5 \%$. 
outro lado, o $\mathrm{P}$ disponível por Mehlich-1 correlacionouse positivamente com o $\mathrm{CO}(\mathrm{r}=0,753 ; \mathrm{p}<0,01)$ e o $\mathrm{P}$ da biomassa microbiana $(r=0,886$; $p<0,01)$. Em solos mais intemperizados, a solubilidade de formas de $\mathrm{P}$ inorgânico (Pi) é muito pequena, e, por esta razão, a disponibilidade de $\mathrm{P}$ seria regulada pela mineralização de Po (Cunha et al., 2007) e pelo fluxo anual de P por meio da biomassa microbiana. Nestes tipos de solos, a biomassa microbiana atuaria como reservatório de $\mathrm{P}$, impedindo que parte do $\mathrm{P}$ incorporado ao solo via mineralização da serapilheira e, ou, adubação mineral se transformasse em formas menos disponíveis para a planta (Matos et al., 2006). Para solos sob plantios de cacau no sul da Bahia, o P disponível correlacionou-se positivamente com todas as frações de Po (total, lábil e microbiano) (Zaia et al., 2006), estimados pelos mesmos métodos do presente trabalho.

Os resultados do presente trabalho evidenciam que a diferença de acúmulo de Po entre as coberturas vegetais seria devida a diferenças de quantidade e qualidade das formas de Po incorporados no solo via os processos de decomposição e mineralização da serapilheira. Pesquisas futuras sobre a qualidade do Po incorporado no solo poderiam esclarecer, em certo limite, por exemplo, por que o solo sob acácia apresentou maior participação do Po na composição do $\mathrm{P}$ total do solo do que na capoeira. Porém, por sua vez, a acácia apresentou o menor teor de P microbiano, que corresponde à fração ativa de Po do solo, e o mais baixo potencial de mineralização de Po do solo. Provavelmente, na acácia, a proporção de formas de Po recalcitrante, especialmente fosfato monoéster, seria maior do que na capoeira. Isto permitiria maior acúmulo de Po na acácia, apesar do menor teor de argila do solo sob essa leguminosa. Assim, parte do $\mathrm{P}$ ciclado na acácia permaneceria na forma de compostos orgânicos, protegidos, de alguma maneira, da competição exercida pela fase mineral do solo. Neste sentido, a maior ciclagem de $\mathrm{C}$ e nutrientes na acácia (Silva, 2005) provavelmente condicionou maior acúmulo de $\mathrm{P}$ na forma orgânica em relação à capoeira.

\section{CONCLUSÕES}

1. Os solos sob plantios de leguminosas florestais apresentaram maior teor ponderado de Po total e Po lábil do que os solos sob pastagem e capoeira.

2. O P disponível correlacionou-se positivamente com o $\mathrm{C}$ e o $\mathrm{P}$ da biomassa microbiana.

3. O Po lábil predominou amplamente sobre a fração inorgânica lábil.

\section{LITERATURA CITADA}

ANDERSON, J.N. \& INGRAM, J.S.I. Tropical soil biology and fertility: A handbook of methods. Wallingford, $\mathrm{CAB}$ International, 1996. 171p.
BARROS, N.F. \& COMERFORD, N.B. Sustentabilidade da produção de florestas plantadas na região tropical. In: ALVAREZ V., V.H.; SCHAFER, C.E.G.R.; BARROS, N.F.; MELLO, J.W.V. \& COSTA, LM., eds. Tópicos em ciência do solo. Viçosa, MG, Sociedade Brasileira de Ciência do Solo, 2002. v.2. p.487-592.

BECK, M.A. \& SANCHES, P.A. Soil phosphorus fraction dynamics during 18 years of cultivation on a Typic Paleudult. Soil Sci. Soc. Am. J, 58:1424-143, 1994.

BOWMAN, R.A. A sequential extraction procedure with concetrated sulfuric acid and diluted base for soil organic phosphorus. Soil Sci. Soc. Am. J., 53:326-366, 1989.

BOWMAN, R.A. \& COLE, C.V. Transformation of organic phophorus substrates in soil as evaluated by $\mathrm{NaHCO}_{3}$ extraction. Soil Sci., 125:95-101, 1978.

BROOKS, P.C.; POWLSON, D.S. \& JENKISON, D.S Phosphorus in the soil microbial biomass. Soil Biol. Biochem., 16:169-175, 1984.

CHEN, C.R.; CONDRON, L.M.; DAVIS, M.R. \& SHERLOCK, R.R. Seasonal changes in soil phosphorus and associated microbial properties under adjacent grassland and forest in New Zealand. For. Ecol. Manag., 177:539-557, 2003.

CANTARELlA, H.; ABREU, C.A. \& BERTON, R.S. Fornecimento de nutrientes pela matéria orgânica do solo. In: GUERRINI, I.A. \& BULL, L.T., eds. Encontros sobre matéria orgânica do solo. Jaboticabal, Universidade Estadual de São Paulo, 1992. p.63-122.

COMERFORD, N.B.; CROPPER, W.; GRIERSON, P.F.; ARAUJO, Q. \& JOSE, S. Modeling P bioavailability and uptake in agroforestry systems. In: GAMA-RODRIGUES, A.C.; BARROS, N.F.; GAMA-RODRIGUES, E.F.; FREITAS, M.S.M.; VIANA, A.P.; JASMIN, J.M.; MARCIANO, C.R. \& CARNEIRO, J.G.A., eds. Sistemas agroflorestais: Bases científicas para o desenvolvimento sustentável. Campos dos Goytacazes, Universidade Estadual do Norte Fluminense, 2006. p.303-315.

CROSS, A.F. \& SCHLESINGER, W.H. A literature review and evaluation of the Hedley fractionation: Aplication to the biogeochemical cycle of soil phosphorus in natural ecosystems. Geoderma, 64:197-214, 1995.

CUNHA, G.M.; GAMA-RODRIGUES, A.C.; COSTA, G.S. \& VELLOSO, A.C.X. Fósforo orgânico em solos sob florestas montanas, pastagens e eucalipto no norte fluminense. $R$. Bras. Ci. Solo, 31:667-671, 2007.

EMPRESA BRASILEIRA DE PESQUISA AGROPECUÁRIA EMBRAPA. Centro Nacional de Pesquisa de Solos. Manual de métodos de análise de solo. 2.ed. Rio de Janeiro, 1997. 212p.

FONTES, A.G. Ciclagem de nutrientes em sistemas agroflorestais de cacau no sul da Bahia. Campos dos Goytacazes, Universidade Estadual do Norte Fluminense, 2006. 71p. (Tese de Doutorado)

GAMA-RODRIGUES, A.C. \& CADIMA-ZEVALLOS, A. Efeito da intensidade de pastejo sobre o sistema radicular de pastagem. Pesq. Agropec. Bras., 26:439-445, 1991

GAMA-RODRIGUES, A.C.; BARROS, N.F. \& MENDONÇA, E.S. Alterações edáficas sob plantios puros e mistos de espécies florestais nativas do sudeste da Bahia, Brasil. R. Bras. Ci. Solo, 23:581-592, 1999. 
GAMA-RODRIGUES, A.C.; BARROS, N.F. \& COMERFORD, N.B. Biomass and nutrient cycling in pure and mixed stands of native tree species in southeastern Bahia, Brazil. R. Bras. Ci. Solo, 31:287-298, 2007.

GEORGE, T.S.; GREGORY, P.J.; ROBINSON, J.R.; BURESH, R.J. \& JAMA, B. Utilization of soil organic P by agroforestry and crop species in the field, western Kenya. Plant Soil, 246:53-63, 2002.

GRESSEL, N. \& McCOLL, J.G. Phosphorus mineralization and organic matter decomposition: A critical review. In: CADISCH, G. \& GILLER, K.E., eds. Driven by nature: Plant litter quality and decomposition. Wallingford, $\mathrm{CAB}$ International, 1997. p.297-309.

GRIERSON, P.F.; COMERFORD, N.B. \& JOKELA, E.J Phosphorus mineralization and microbial biomass in a Florida Spodosol: Effects of water potential, temperature and fertilizer application. Biol. Fert. Soils, 28:244-252, 1999.

GRIERSON, P.F.; SMITHSON, P.; NZIGUHEBA, G.; RADERSMA, S. \& COMERFORD, N.B. Phosphorus dynamics and mobilization by plants. In: NOORDWISK, M.; van. CADISCH, G. \& ONG, C.K., eds. Below-ground interactions in tropical agroecosystems: Concepts and models with multiple plant components. Wallingford, $\mathrm{CAB}$ International, 2004. p.127-142.

GUERRA, J.G.M.; FONSECA, M.C.C.; ALMEIDA, D.J.; DEPOLLI, H. \& FERNANDES, M.S. Conteúdo de fósforo em amostras de solos. Pesq. Agropec. Bras., 31:291-299, 1996.

LEHMANN, J.; GÜNTHER, D.; MOTA, M.S.; ALMEIDA, M.P. ZECH, W. \& KAISER, K. Inorganic and organic soil phosphorus and sulfur pools in an Amazonian multistrata agroforestry system. Agrofor. Syst., 53:113-124, 2001.

LUGO, A.E.; CUEVAS, E. \& SANCHEZ, M.J. Nutrients and mass in litter and soil of ten tropical tree plantations. Plant Soil, 125:263-280, 1990.
MATOS, E.S.; MENDONÇA, E.S.; VILLANI, E.M.A.; LEITE, L.F.C. \& GALVÃO, J.C.C. Formas de fósforo no solo em sistemas de milho exclusivo e consorciado com feijão sob adubação orgânica e mineral. R. Bras. Ci. Solo, 30:625632,2006 .

MURPHY, J. \& RILEY, J.P. A modified single solution method for the determination of phosphate in natural waters. Anal. Chem. Acta, 27:31-36, 1962.

NOVAIS, R.F.\& SMYTH, T.J. Fósforo em solo e planta em condições tropicais. Viçosa, MG, Universidade Federal de Viçosa, 1999. 399p.

SILVA, M.P.S. Biomassa e caracterização química da serapilheira e do solo sob diferentes coberturas florestais na região norte fluminense. Campos dos Goytacazes, Universidade Estadual do Norte Fluminense, 2005. 16p. (Monografia - Graduação em Agronomia)

SIQUEIRA, J.O. \& MOREIRA, F.M.S. Biologia e bioquímica do solo. Lavras, UFLA/FAEPE, 2001. 291p.

SOLOMON, D.; LEHMANN, J.; MAMO, T.; FRITZCHE, F. \& ZECH, W. Phosphorus forms and dynamics as influenced by land use changes in the sub-humid Ethiopian highlands. Geoderma, 105:21-48, 2002.

SZOTT, L.T. \& MELENDEZ, G. Phosphorus availability under annual cropping, alley cropping, and multistrata agroforestry systems. Agrofor. Syst., 53:125-1132, 2001.

TIESSEN, H.; STEWART, J.W.B. \& COLE, C.V. Pathways of phosphorus transformation in soils of differing pedogenesis. Soil Sci., 48:853-858, 1984.

VANCE, E.D.; BROOKES, P.C. \& JENKINSON, D.S. An extraction method for measuring soil microbial biomass C. Soil Biol. Biochem., 19:703-707, 1987.

ZAIA, F.C.; GAMA-RODRIGUES, A.C.; GAMA-RODRIGUES, E.F. \& MACHADO, R.C.R. Organic phosphorus variation in soils from different cacao plantations in south of Bahia, Brazil. In: INTERNATIONAL COCOA RESEARCH CONFERENCE, 15., San Jose, 2006. Proceedings. San Jose, Cocoa Producers' Alliance, 2006. p.1-11. CD-ROM. 